\title{
Comprometimento cognitivo e as práticas de autocuidado entre diabéticos tipo 2 do Programa de Automonitoramento Glicêmico de Clínica Escola de Enfermagem
}

\author{
Vanusa dos Santos*, Ioná Bez Birolo Vieira, M.Sc.**, Luciane Bisognin Ceretta***, \\ Cristiane Damiani Tomasi****
}

${ }^{*}$ Graduada em enfermagem pela UNESC (Universidade do Extremo Sul Catarinense) - Criciúma/SC, **Docente do Curso de Enfermagem - UNESC, ***Doutoranda em Ciências da Saúde - Diretora da Unidade Acadêmica da Saúde (UNASAU) UNESC, ****Doutoranda em Enfermagem - Docente do Curso de Enfermagem - UNESC

\section{Resumo}

Este estudo objetivou identificar a prevalência de comprometimento cognitivo entre os pacientes diabéticos tipo 2. Trata-se de uma pesquisa quantitativa que utilizou para a coleta de dados a entrevista estruturada e o Mini Exame do Estado Mental, e os dados foram analisados por meio do programa SPSS 17.0. Foi realizada, em uma Clínica Escola de Enfermagem, com 85 pacientes cadastrados no Programa de Automonitoramento Glicêmico, no período de janeiro a março de 2011. Nos resultados, comprovou-se o comprometimento cognitivo entre diabéticos tipo 2, causando preocupaçáo, pois a adesão ao tratamento medicamentoso e as práticas de autocuidado podem estar prejudicadas, embora a maioria dos pacientes relatassem a realização do autocuidado adequadamente.

Palavras-chave: cognição, autocuidado, diabetes mellitus.

\section{Abstract \\ Cognitive commitment and self-care practices among patients with diabetes II of Glucose Self-monitoring Program of a Clinic Nursing School}

This study aimed at identifying the prevalence of cognitive impairment among patients with diabetes type 2 . It was a quantitative structured interview which used the Mini Mental State Examination to collect data and, then, were analyzed using SPSS 17.0. It was conducted in a Clinic Nursing School with 85 patients registered in the Glucose Self-monitoring Program from January to March 2011. The results showed cognitive impairment among patients with diabetes type 2, causing concern due to adherence to drug treatment and self-care practices may be compromised, although most patients reported performing self-care properly.

Key-words: cognition, self-care, diabetes mellitus. 


\section{Resumen}

\section{Deterioro cognitivo y las prácticas de autocuidado entre pacientes diabéticos tipo 2 del Programa de Automonitoreo de la Glucosa de Escuela Universitaria de Enfermería}

Este estudio tuvo como objetivo identificar la prevalencia del deterioro cognitivo en pacientes con diabetes tipo 2. Se trata de una investigación cuantitativa que utilizó para la recolección de datos la entrevista estructurada y el Mini Examen del Estado Mental, y después fueron analizados utilizando el programa SPSS 17.0. Se realizó en la Escuela Universitaria de Enfermería con 85 pacientes registrados en el Programa de Automonitoreo de la Glucosa desde enero a marzo de 2011. Los resultados mostraron el deterioro cognitivo en pacientes con diabetes tipo 2, causando preocupación, pues la adherencia al tratamiento farmacológico y las prácticas de autocuidado pueden verse comprometidos, aunque la mayoría de los pacientes informaron que realizaban el autocuidado correctamente.

Palabras-clave: cognición, autocuidado, diabetes mellitus.

\section{Introdução}

O Diabetes Melitus (DM) é uma epidemia que está em curso, sendo que em 1985 estimava-se que existissem 30 milhóes de diabéticos, em 1995 esse número aumentou para 135 milhóes, atingindo 173 milhôes em 2002, com projeção de alcançar os 300 milhóes no ano 2030, sendo que nos países desenvolvidos esta epidemia é mais intensa [1].

Com o avanço desta doença, desperta a necessidade de uma ampla abordagem sobre o DM e suas complicaçóes, por isso um dos objetivos da educação em saúde é prevenir o avanço destas estimativas.

As complicaçôes decorrentes do DM representam um significante problema de saúde pública, considerando que o tratamento destas exige tecnologias de alto custo, sobrecarregando o sistema de saúde, necessitando de novas ferramentas que visem prevenir ou retardar tais complicaçóes ou, ainda mais que isto, prevenir o desenvolvimento do DM. Dentre as principais complicações estão: a insuficiência renal, amputação de membros inferiores, cegueira, doenças cardiovasculares, elevada morbimortalidade e o comprometimento da qualidade de vida do portador da doença [2].

A Organização Mundial da Saúde (OMS) estimou em 1997 que, após 15 anos de doença, 2\% dos indivíduos acometidos estarão cegos e $10 \%$ terão deficiência visual grave. Além disso, estimou que, no mesmo período de doença, 30 a 45\% terão algum grau de retinopatia, 10 a $20 \%$ de nefropatia, 20 a
$35 \%$ de neuropatia e 10 a $25 \%$ terão desenvolvido doença cardiovascular [3].

Outra questáo importante que suscita a necessidade da investigação são as práticas de autocuidado adotadas pelos pacientes diabéticos (usar calçados fechados e confortáveis, secar entre os dedos dos pés, não andar descalços, manter as unhas dos pés curtas, etc.) uma vez que estas práticas quando corretamente adotadas, podem ser importantes fatores protetores contra as complicaçôes da doença, sempre que o portador de DM tenha capacidade cognitiva para inserir em sua rotina de vida tais práticas.

Conforme Xavier [4], na prática assistencial, é possível verificar o impacto que o DM possui sobre a saúde da população e também as dificuldades de adesáo dos portadores aos comportamentos preventivos. A partir deste contexto, o enfermeiro, enquanto profissional de saúde engajado na assistência ao diabético, deve obter novas estratégias para a prática de autocuidado promovendo a saúde do paciente, já que a adesão ao tratamento e as práticas de autocuidado são pontos frágeis da educação em saúde e que, portanto, merecem ser refletidos profundamente.

Este estudo buscou evidenciar a prevalência do comprometimento cognitivo entre diabéticos que usam insulina e as práticas de autocuidado adotadas por estes pacientes.

\section{Material e métodos}

A abordagem metodológica apresentada é de caráter quantitativo, do tipo descritivo. A pesquisa 
com abordagem quantitativa é utilizada somente quando o instrumento de medida utilizável e válido, desejando garantir a objetividade e credibilidade dos achados, sempre que o instrumento não coloque em risco a vida humana [5].

Conforme Parras Filho [6], observa, registra, analisa e correlaciona fatos ou fenômenos sem manipulá-los, desvendando assim com precisão a frequência com que ocorre determinado fenômeno, utilizando como instrumento a observação, a entrevista e o questionário.

Vale ressaltar que as vantagens desses estudos são várias: têm custo relativamente baixo, são fáceis de serem conduzidos e não demoram muito tempo para serem concluídos, o que compensa, de certa forma, a dificuldade da interpretação [7].

A pesquisa foi realizada na Clínica Escola do Extremo Sul Catarinense, participando da pesquisa 85 pacientes cadastrados no programa de automonitoramento no período de janeiro a março de 2011. Todos os pacientes incluídos no estudo assinaram o Termo de Consentimento Livre e Esclarecido. O projeto teve aprovação do CEP da instituição hospitalar com parecer número 338/2010.

Os dados foram analisados pelo programa Software Statistical Packagefor the Sciences (SPSS) 17.0 existente no laboratório de epidemiologia da Unidade Acadêmica de Ciências da Saúde (UNASAU). Logo os gráficos e tabela foram elaborados no Excel. Os resultados foram apresentados em tabelas e gráficos, interpretados e discutidos a luz das referências disponíveis sobre o assunto.

\section{Resultados e discussão}

Apresentaremos a seguir a análise e os resultados obtidos através da coleta de dados, no qual se pode verificar o perfil sociodemográfico dos pacientes pesquisados, a caracterização quanto à patologia, à adesão ao tratamento medicamentoso e à prevalência de comprometimento cognitivo entre os pacientes com diabetes mellitus tipo 2 (DM2) atendidos na CEE.

Em relação ao gênero, dos 85 pacientes pesquisados, 47 eram do sexo feminino e 38 masculino. A faixa etária que predominou no gênero masculino foi de 50 a 59 anos com $50 \%(\mathrm{n}=19)$, e no gênero feminino foi de 60 a 69 anos com 46,8\% ( $=22)$, enquanto que $63,9 \%(n=30)$ e $31,6 \%(n=12)$ feminino e masculino, respectivamente, correspon- dem ao percentual de idosos, classificados conforme o estatuto do idoso.

Com base nestes dados, salientamos que o envelhecimento da população é um dos maiores triunfos da humanidade e também um dos nossos grandes desafios, pois com o envelhecimento natural, algumas capacidades cognitivas diminuem naturalmente, assim como sua capacidade de realizar atividades de vida diária, o autocuidado e a adesão ao tratamento medicamentoso [8].

Quanto ao estado civil, observa-se que a predominância de pacientes casados em ambos os gêneros, correspondendo $84,2 \%(\mathrm{n}=32)$ dos homens e $53,2 \%(n=25)$ das mulheres. O índice de mulheres viúvas e divorciadas também foi considerável, correspondendo a $25,5 \%(\mathrm{n}=12)$ e $12,8 \%(n=6)$, respectivamente, tornando assim um fator preocupante devido à ausência de um companheiro para as mesmas, pois a faixa etária mais atingida pela DM2 ficou entre 60 e 69 anos, sendo classificadas como idosas, e sabe-se que os idosos apresentavam diminuição significativa de memória e cognição. Logo as mulheres que são divorciadas e viúvas, dependendo da organização familiar podem ficar desamparadas nos quesitos: autocuidado, adesão ao tratamento e conhecimento de possíveis complicaçóes que podem aparecer se náo houver um controle glicêmico.

Com relação à ocupação dos pacientes, observa-se que $55,3 \%(\mathrm{n}=21)$ dos pacientes do sexo masculino eram aposentados, não muito diferente das pacientes do sexo feminino, pois $46,8 \%(\mathrm{n}=22)$ também eram aposentadas. Porém vale ressaltar que os aposentados com outra ocupação e os beneficiários aumentaram a porcentagem de previdenciários, pois ambos também recebem verba da previdência social, deste modo, o percentual corresponderia a $76,4 \%(n=29)$ e $68,1 \%(\mathrm{n}=32)$.

Os pacientes com diagnóstico DM no período de até 5 anos, $24,1 \%(\mathrm{n}=13)$ faziam uso somente da insulina NPH (Neutral Protamine Hagedorn), considerada como insulina basal, que cobre as necessidades de insulina entre as refeiçôes, bloqueando a gliconeogênese hepática. Com o mesmo tempo de diagnóstico faziam uso de ambos os tipos (Regular e NPH) 17,2\% ( $\mathrm{n}=$ 5), sendo que a insulina regular é considerada prandial, administrada para cobrir as necessidades de insulina nas refeiçóes, diminuindo os níveis glicêmicos com mais rapidez [1]. 
Assim, os pacientes que obtiveram diagnóstico de DM entre 6 e 15 anos, percebeu-se um aumento significativo no uso das insulinas $\mathrm{NPH}$ e regular, correspondendo a $50 \%(\mathrm{n}=27)$, no uso de insulina $\mathrm{NPH}$, e 58,6\% ( $\mathrm{n}=7)$ no uso de ambos os tipos de insulina. Os pacientes com diagnóstico DM entre 16 e 25 anos náo demonstraram nenhuma diferença quanto ao uso do tipo de insulina. Os pacientes com diagnóstico acima de 6 anos apresentaram altos índices de sobrepeso, obesidade e obesidade grave, considerando que os pacientes que possuem tais classificaçóes possuem uma redução da sensibilidade da insulina, sendo necessário aumentar a dosagem das insulinas NPH e regular para compensar o excesso de glicose.

Com relação à aplicação da insulina, verificou-se que $85,7 \%(n=42)$ náo realizaram rodízio, ou seja, aplicavam somente na regiáo umbilical devido à sensibilidade, pois relataram que este local causa menos dor; 36,1\% $(\mathrm{n}=13)$ dos pacientes que realizavam o rodízio alternavam entre a regiáo umbilical e o braço; e $25 \%(\mathrm{n}=9)$ dos pacientes seguiam o rodízio conforme as orientaçóes recebidas pelos profissionais da saúde. Segundo o MS, a aplicação deve ser debaixo da pele, ou seja, subcutânea, sempre alternando os locais de aplicação a fim de que se possam evitar as complicaçóes tais como hipertrofia ou atrofia do local, sendo que os locais indicados são braços (na parte externa e superior), nas coxas (parte anterior e lateral), regiáo abdominal e regiáo glútea.

A partir desta problemática é de suma importância a intervenção do profissional enfermeiro nas orientaçóes quanto aos locais de aplicação, e a importância do rodízio como prevenção de possíveis complicaçôes nesses locais.

Com relação à frequência de aferição da glicemia, observa-se que dos pacientes diagnosticados em até 2 anos, 69,8\% (n = 44) aferiam a glicemia 2 vezes ao dia, náo sendo diferente nos pacientes com diagnóstico entre 3 e 5 anos, que também prevaleceu a aferição de 2 vezes ao dia, correspondendo a 53,3\% $(\mathrm{n}=8)$. Os pacientes que foram diagnosticados entre 6 e 10 anos aferiam 3 vezes por dia, correspondendo a $60 \%(n=3)$.

Acredita-se que os pacientes que aferem a glicemia 2 vezes ao dia mantêm os níveis glicêmicos sob controle, pois conforme a OMS, vários estudos conduzidos até o momento demonstraram que mudanças no estilo de vida podem favorecer o controle de muitas complicaçóes, sendo desnecessário o aumento na frequência da aferição [8].

Grande parte dos pesquisados realizavam o autocuidado com os pés e também possuía conhecimento sobre a importância de verificar diariamente os pés para evitar maiores complicaçóes, pois se acredita que os cuidados por eles adotados são devido ao tempo que os mesmos convivem com a doença, o que os fazem realizar rotineiramente. Conforme relato dos pacientes que não realizavam o cuidado, afirmaram possuir conhecimento sobre os benefícios, porém não os realizavam por falta de tempo ou por esquecimento.

Conforme a OMS, o pé diabético é definido como a "situação de infecção, ulceração ou também destruiçáo dos tecidos profundos dos pés, associado a anormalidades neurológicas e vários graus de doença vascular periférica nos membros inferiores de pacientes com diabetes mellitus [2]".

É importante a atuação do enfermeiro juntamente com sua equipe de saúde na orientação aos pacientes diabéticos sobre os cuidados diários com os pés e na prevenção das úlceras [9]. Cabe ao enfermeiro realizar a inspeção dos pés, verificando a textura, coloração, hidratação, presença de rachaduras, micose interdigital, lesóes na pele, verificar os pulsos periféricos, e também realizar exame neurológico, a fim de averiguar a sensibilidade tátil, térmica e dolorosa, pois o pé diabético requer tratamento e acompanhamento constante, pois possui uma cicatrizaçáo longa, exigindo cuidados e curativos frequentes, no entanto a educação do paciente, juntamente com a assistência da enfermagem, é essencial para obter bons resultados, e também prevenir novas lesóes [2].

Vale ressaltar que na amostra deste estudo nenhum paciente apresentou pé diabético como complicação do diabetes. Conforme o consenso internacional sobre pé diabético, esta complicação representa uma das mais incapacitantes complicaçóes crônicas advindas do mau controle da doença, com impacto social e econômico para as famílias, o sistema de saúde e a sociedade, isto tanto em países desenvolvidos como emergentes [10].

No conhecimento das complicaçóes, prevaleceu o comprometimento com os olhos, rins e pés. A OMS afirma que "O diabetes é a principal causa de cegueira, amputaçóes e disfunçóes renais. Estas complicaçôes são responsáveis por grande parte do custo social e financeiro do diabetes [8]". 
O desconhecimento da hipertensão enquanto complicação do DM aparece em 51,2\% ( $\mathrm{n}=42)$ da amostra, despertando a necessidade de uma abordagem sistêmica que relacione a abordagem das duas patologias, para melhor compreensão dos pacientes,

É de competência dos profissionais de saúde promover a educaçáo auxiliando os diabéticos a conviver com as possíveis complicaçóes, promovendo conhecimentos e habilidades para o autocuidado.

Os pacientes diabéticos utilizavam várias classes medicamentosas, mas destacou-se o uso dos anti-hipertensivos juntamente com antidiabéticos orais, correspondendo a 74,1\% ( $\mathrm{n}=$ $60)$, logo em seguida estava o uso de antiplaquetários com 59,3\% ( $\mathrm{n}=48)$. O DM2 pode provocar ao longo do tempo a descompensação da glicemia, aumentando a quantidade de medicamentos, contribuindo para uma situaçáo de polifarmácia. Esta patologia proporciona ao portador maiores chances de desenvolvimento de outras patologias e complicaçóes, promovendo o aumento do número de fármacos utilizados pelos pacientes [11]. Acredita-se que o desenvolvimento de outras patologias se dê pelo estresse causado pela mudança de vida que ocorre para o controle do DM.

Quanto a quantidade de medicamento administrado, $89 \%(\mathrm{n}=76)$ dos pesquisados fazem uso de mais de 4 tipos de medicamentos. A partir destes dados, desperta a preocupaçáo de que o uso de polifarmácia pode estar agravando ainda mais a sua saúde, uma vez que a polifarmácia está associada ao aumento da gravidade das reaçóes adversas, aumento das interaçóes medicamentosas, causando toxicidade acumulativa e consequentemente redução na adesão ao tratamento e elevação da morbimortalidade [12].

Segundo Nascimento et al. "[...] não é a quantidade de medicamentos administrados diariamente, tampouco o uso de insulina exógena, que se configura como agente estressor, mas, sim, a frequência de tomadas diárias de medicamentos [13]".

Conforme relatos dos pacientes, 95\% $(\mathrm{n}=$ 81) eram aderentes ao tratamento, considerando que estes valores foram obtidos por meio da aplicaçáo da escala de MAT. No momento da pesquisa ao responder as indagaçóes da escala de MAT, os pacientes se autodefiniram como aderentes por achar que estavam tomando corretamente as medicaçôes.

O termo adesão é definido, como a utilização dos medicamentos prescritos ou outros procedimentos em pelo menos $80 \%$ de seu total, observando horários, doses e tempo de tratamento [14]. No entanto os pacientes relatavam que não mantinham um rigor no horário na administração da medicação, mas sempre conforme prescrição médica.

$\mathrm{O}$ fato de os pacientes serem na maioria idosos também pode ter influenciado nos dados encontrados, pois a diminuição da atenção do idoso, sua memória de trabalho e capacidade de planejamento podem deixá-lo confuso e achar que já tomou a medicação ou esquecer-se de tomá-la [11].

A partir dos dados da pesquisa, observou-se que $85 \%(\mathrm{n}=72)$ dos pacientes náo participavam dos grupos de orientação nos bairros, sendo preocupante, pois se entende que o fato de compartilhar situaçóes e problemas semelhantes contribui para uma convivência de alto valor terapêutico, pois o paciente pode verbalizar barreiras pessoais e sociais, relatando suas experiências e sugestóes sobre os problemas vivenciados e isso certamente facilitará na adesão ao tratamento [15]. O convívio com pessoas que possuem os mesmo problemas e dúvidas além de proporcionar solidariedade mútua entre os participantes promove também o incentivo para a promoção do autocuidado e aceitaçáo da doença e suas complicaçóes.

No entanto 52,7\% ( $\mathrm{n}=44)$ dos pacientes relataram não participar dos grupos de orientaçóes por falta de oportunidade, participando apenas da entrega das medicaçóes mensalmente. Por outro lado, os profissionais enfermeiros possuem papel fundamental na atenção em saúde, especialmente no que se refere às açôes educativas com a população, utilizando este instrumento como estratégia de promoção em saúde, cabendo a ele promover mudanças para sanar as necessidades apresentadas [16].

Através da aplicação do Mini Exame do Estado Mental, observou-se que 19\% (n = 16) dos pacientes possuíam comprometimento cognitivo, sendo que 05 destes pacientes têm menos de 60 anos e, 11 acima de 60 anos. A partir dos dados podemos verificar que a maioria dos pacientes que apresentaram comprometimento cognitivo eram idosos. Dos pacientes com comprometimento cognitivo $27 \%(n=4)$ eram analfabetos e/ou com 
baixa acuidade visual, enquanto que $73 \%(n=12)$ eram alfabetizados.

Estes dados são bastante relevantes, uma vez que estando com as propriedades cognitivas comprometidas, a adesão ao tratamento e as medidas de autocuidado também se comprometem. O comprometimento cognitivo afeta consideravelmente a autonomia do paciente frente as suas necessidades, tornando-se assim, um agravante para qualquer tipo de doença [17].

Os pacientes associam que, após um determinado tempo do diagnóstico, intensifica-se a diminuição da memória, principalmente em períodos que não conseguem manter a glicemia nos níveis adequados.

Dos pacientes que apresentaram comprometimento cognitivo, $82,7 \%(\mathrm{n}=14)$ não eram aderentes ao tratamento medicamentoso. Os dados sugerem que se o paciente fosse aderente ao tratamento e conseguisse manter a glicose dentro dos parâmetros desejáveis, também se conseguiria minimizar as complicaçóes da doença, dentre elas a cognição do paciente. Quanto à realização da atividade física, observou-se que 93,8\% ( $\mathrm{n}=15)$ não realizavam a mesma, porém, acredita-se que a realização regular da mesma, pode diminuir o comprometimento cognitivo ou ao menos minimizar seus efeitos da vida diária dos pacientes diabéticos.

Verificou-se que $81,3 \%(\mathrm{n}=13)$, que realizavam a insulinoterapia há 5 anos, apresentaram comprometimento cognitivo. Conforme os dados percebe-se que o tempo de insulinoterapia náo influencia do déficit cognitivo, pois quanto maior o tempo de uso, menor a incidência deste. No entanto, surge a hipótese que o início do tratamento cause estresse o suficiente para que desenvolva algum comprometimento cognitivo, já que a pesquisa demonstrou um percentual significativo entre os primeiros anos de uso da insulina.

No que se refere à participação nos grupos terapêuticos, observou-se que 93,8\% $(n=15)$ não participavam dos grupos de orientação, e somente $6,3 \%(n=1)$ participavam dos grupos. A participação nos grupos de orientação proporciona ao paciente oportunidade de compartilhar suas dúvidas e anseios com outras pessoas que possuem o mesmo problema, adquirindo mais conhecimento e podendo assim melhorar suas práticas de autocuidado.

\section{Conclusão}

A partir desta pesquisa pôde-se obter o conhecimento da existência de comprometimento cognitivo nos pacientes diabéticos e quais os fatores que podem influenciar no desenvolvimento da mesma, pois se observou que os pacientes não adotam algumas práticas que podem amenizar o comprometimento cognitivo, assim cabe aos profissionais buscar estratégias que motivem os pacientes diabéticos a adotar tais práticas.

O diabetes melittus é uma doença multifatorial e de abordagem complexa o que desperta o interesse de aprofundar suas demandas, podendo ser considerada uma fonte inesgotável de conhecimento e saberes para quem pesquisa. A enfermagem enquanto profissão engajada no cuidado deve obter maiores conhecimentos dessa doença, para promover as práticas necessárias e fortalecer a integralidade do cuidado aos pacientes com DM.

\section{Referências}

1. Sociedade Brasileira de Diabetes. Tratamento e acompanhamento do diabetes mellitus: diretrizes da Sociedade Brasileira de Diabetes. Sáo Paulo: SBD; 2007.

2. Manual de Enfermagem. Cuidados de enfermagem em diabetes mellitus. São Paulo: Sociedade Brasileira de Diabetes; 2009. 171p.

3. Ministério da Saúde. Secretaria de Atenção à Saúde. Departamento de Atenção Básica. Diabetes Mellitus. Brasília: Ministério da Saúde; 2006. p.64.

4. Xavier A, Bittar D, Ataíde M. Crenças no autocuidado em diabéticos- Implicações para a prática. Texto Contexto Enferm 2009;18(1):124-30.

5. Leopardi MT. Metodologia da pesquisa na saúde. Santa Maria: Pallotti; 2001. p. 344.

6. Parra Filho D, Santos JA. Metodologia científica. São Paulo: Futura; 2000. 277p.

7. Vieira S, Ramos IC. Prevalência de pé diabético e fatores associados nas unidades de saúde da família da cidade do Recife, Pernambuco, Brasil, em 2005. Cad Saúde Pública 2008;24(12):2861-70.

8. World Health Organization. Envelhecimento ativo: uma política de saúde. Brasília: Organização Pan-Americana da Saúde; 2005.

9. Hirota CMO, Haddad MCL, Guariente MHDM. Pé diabético: $O$ papel do enfermeiro no contexto das inovaçóes terapêuticas. Ciência Cuidado e Saúde 2008;7(1):114-20. 
10. Rocha CH, Oliveira APS, Ferreira C, Faggiani FT, Schroeter G, Souza Araújo ACA, et al. Adesão à prescrição médica em idosos de Porto Alegre, RS. Ciênc Saúde Coletiva 2008;13(suppl)703-710.

11. Trentin CSN. Adesão medicamentosa em pacientes idosos diabéticos [Dissertação]. Porto Alegre: PUCRS; 2009.

12. Secoli SR. Polifarmácia: Interações e reações adversas no uso de medicamentos por idosos. Rev Bras Enferm 2010;63(1):136-140.

13. Nascimento AB, Chaves EC, Grossi SAA, Lottenberg SA. A relação entre polifarmácia, complicaçôes crônicas e depressão em portadores de Diabetes Mellitus Tipo 2. Rev Esc Enferm USP 2010;44(1):40-6.
14. Leite SN, Vasconcellos MPC. Adesão à terapêutica medicamentosa: elementos para a discussão de conceitos e pressupostos adotados na literatura. Ciênc Saúde Coletiva 2003;8(4):775-82.

15. Fernandes JOS. Idosos diabéticos. Acompanhamento de um grupo de diabéticos em uma unidade básica de saúde - PUCSP [Dissertaçáo]. Sáo Paulo: Pontifícia Universidade Católica de São Paulo; 2006.

16. Donaduzzi JC. Açóes Educativas de enfermeira em estratégia de saúde da família [Dissertação]. Santa Maria: Universidade Federal de Santa Maria, Centro de Ciências da Saúde; 2009.

17. Texeira JJ, Lefèvre F. A prescriçẫo medicamentosa sob a ótica do paciente idoso. Rev Saúde Pública 2001;35(2):207-13. 